\title{
SECONDARY SYPHILITIC INFECTION *
}

\author{
CHARLES MALLORY WILLIAMS, M.D. \\ Associate Professor of Dermatology, Post-Graduate Medical School; Instructor \\ in Dermatology, University and Bellevue Medical College; Attend- \\ ing Physician, New York Skin and Cancer Hospital \\ NEW YORK
}

The disinclination to admit the possibility of a second infection with syphilis in a patient in whom the acquired disease has become generalized is fortified by clinical experience of many years. A few doubtful cases might be cited, but the preponderant weight of the opinion of the medical profession has been against such an interpretation of the facts presented. We are still ignorant of the explanation of the resistance of the tissues of a syphilitic patient to infection from without, while susceptible to local infection from within, especially as some of the late genital lesions often closely simulate a chancre.

If there have been so few, if any, authentic cases of reinfection, the explanation appears to be simple-there were few cases of radical cure. Some of the greatest authorities, notably Fournier and Hutchinson, believed, apparently, that they could eradicate the disease, and they certainly were able to follow some of their patients for many years and to see them remain apparently well themselves, and become the parents of healthy children. In view, however, of our present knowledge of the persistence of small foci of infection in the heart, aorta and other organs, with no clinical signs except a positive Wassermann reaction, we are justified in doubting the completeness of these cures. If it is true that the absence of cases of reinfection is due to the absence of cases of radical cure, it follows that if radical cure can be obtained, a certain number of cases of reinfection should occur. It may be, on the other hand, that the rarity or absence of cases of reinfection is due to a partial immunity which persists after the extinction of the infectious process, as in typhoid fever and in the exanthems. If this were true, a greater number of radical cures would not be followed by an increased number of cases of reinfection. The improvement in treatment which has occurred in the past ten years has been accompanied by a great increase in the number of reported and apparently authentic cases of reinfection, and it is increasingly probable, therefore, that resistance to reinfection is due to the presence of the disease itself, and not to an enduring immunity. \section{College.}

*From the Dermatological Service, University and Bellevue Medical 
The possibility that a patient with congenital syphilis may subsequently acquire the disease has never, so far as I know, been questioned. Indeed, this occurrence is constantly invoked to explain the apparent cases of syphilis of the third generation. Why should we admit the possibility of reinfection in a congenital syphilitic patient and deny such a possibility in a victim of the acquired disease? It would seem to be more reasonable to assume that in both cases the possibility depends on the extinction of the infection either by treatment or by the lapse of time. The question is: Do such reinfections occur? As absolute proof is difficult or impossible to obtain, we must decide by the balance of probability, and approach the question with an open mind. The following case, which occurred in the service of Prof. William B. Trimble at the University and Bellevue dispensary, is most easily explained, I believe, on the theory of reinfection.

\section{REPORT OF A CASE}

History.-Michael G., aged 27, when first seen, Aug. 9, 1916, gave a history of penile sore eight months previously, for which he had been treated with about twenty injections of mercury. He complained of headaches and pains in the extremities, and showed extensive mucous patches on both tonsils. The Wassermann reaction was ++++ .

Treatment.-Nine-tenths gram of neo-arsphenamin was administered Aug. 11 and Nov. 3, 10, 17 and 24, 1916. Nine-tenths gram of arsphenamin was administered July 6 and $0.6 \mathrm{gm}$. on July 20 and Aug. 3, 1917. Six-tenths gram of arsphenamin was administered March 1, 8, 15 and 22, 1918. Mercury in the form of intramuscular injections of the salicylate and mixed treatment internally, was given throughout the course, but the patient was a sailor, and the treatment was necessarily irregular. All symptoms disappeared under treatment. Wassermann Reaction: After the first test, this varied from negative to + , except on Feb. 18, 1918, when it was ++ . Sept. 14, 1918, the reaction was negative, and three tests made in the army between Sept. and Dec. 15, 1918, were said to be negative.

Second Occurrence of Condition.-Nov. 19, 1919, the patient returned with a penile sore of four weeks duration, occurring on the site of the original primary lesion. The incubation period could not be established, as he had been exposed several times in the month preceding the appearance of the sore. The border was firm and the base indurated. The inguinal glands were enlarged. The Wassermann reaction was negative November 19, and again on December 3. Dark-field examination made November 19 revealed no spirochetes, presumably on account of the use of an antiseptic dressing. Microscopic examination on December 12 revealed both Spirochaeta pallida and the Ducrey bacillus.

\section{DIFFERENTIAL DIAGNOSIS}

In this case the diagnosis in the first instance was made both by clinical and by laboratory methods; treatment by both arsphenamin and mercury was faithfully, if somewhat irregularly, carried out; all symptoms-both clinical and serologic-disappeared; and although the 
Wassermann reaction at the time was negative, exposure was followed by the development of a sore in which spirochetes were demonstrated. If this sore was not a chancre, what was it? Under the title "chancre redux," Fournier ${ }^{1}$ describes a lesion clinically indistinguishable from a chancre, occurring on the site of a previous chancre, usually within a week or two of the healing of the first. It is in reality a fresh ulceration in the induration remaining from the original sore. The case under discussion cannot fall in this category as the induration of the original sore had entirely disappeared, and the interval was two years instead of a few weeks.

Under the same title, "chancre redux," or "recurring chancre," Hutchinson ${ }^{2}$ describes a lesion exactly resembling a chancre, which may occur after an interval of from a few months to several years, and which almost invariably occurs in the site of the primary lesion. He states that it is not attended by glandular enlargement and is not contagious, and adds: "Whether the cartilaginous induration should be classified as gummata is a question chiefly of definitions." Some of the cases were accompanied by tertiary symptoms in other parts of the body. The lesion described by Hutchinson as "chancre redux" had previously been described by Fournier ${ }^{3}$ as the pseudo-chancre induré, and the identity of the two is accepted by Hutchinson. The interval after the first sore varied from ten months to several years. Fournier insists that clinically, as far as the sores themselves are concerned, the chancre and the pseudo-chancre are indistinguishable, and his statement must be accepted as true. He agrees with Hutchinson that the satellite glands are seldom involved, but describes three exceptional cases among the twenty-one which form the basis of his report, in which such adenopathy occurred. In several cases there were other symptoms of syphilis present. Fournier states that the pseudo-chancre frequently occurs in the scar of the primary lesions, but he does not assert that this location is invariable. He gives the histories of three cases, in one of which the pseudo-chancre occurred on a different part of the penis, in another on the upper lip, and in the third on the scrotum. From the point of view of diagnosis, it therefore appears to be immaterial whether the two lesions involve the same area or not. These lesions are distinctly tertiary in type; that is, they are solitary localized ulcers, with little or no tendency toward glandular enlargement, and, according to Hutchinson, they are noncontagious. Fournier cites one case in

1. Fournier, A.: Etude clinique sur l'induration syphilitique primitive, Arch. gen. de méd., November, 1867.

2. Hutchinson: Syphilis, London, 1909, p. 171.

3. Fournier, A.: Du pseudo chancre induré des sujets syphilitiques, Arch. gen. de méd., June, 1868. 
which the wife of the patient remained free of all symptoms of syphilis; he does not mention this point in regard to the others. This absence of contagiousness is to be expected, as it is usually the rule in late lesions.

It is possible that the case under discussion belongs in this group; absolute proof to the contrary is impossible. The case differs, however, in many important respects: First, the patient had received more vigorous treatment than either Fournier or Hutchinson were acquainted with. Second, he had no other symptoms of syphilis, as was true in some of the cases of pseudo-chancre; also, the Wassermann reaction was persistently negative. Third, the sore was infectious, as was shown by the presence of spirochetes. Fourth, the neighboring glands were enlarged and painless.

Of course, a tertiary lesion may simulate exactly the clinical appearance of a chancre; it may coexist with a persistent negative Wassermann reaction; it may be contagious, although the demonstration of spirochetes is rarely possible; and it may be accompanied by a satellite adenopathy; but all these unusual phenomena seldom occur in the same patient at the same time. It is more reasonable to believe that the man was cured and was reinfected. If the possibility of a cure is admitted, the case presents no difficulty.

\section{CASES OF REINFECTION REPORTED IN THE LITERATURE}

Within the last few years, the number of reported cases of reinfection has increased rapidly. Benario's ${ }^{4}$ monograph, published in 1914, gives complete details of ninety-six cases collected from the literature, of which forty-one satisfy the requirements which he considers necessary to establish a diagnosis of reinfection. Of the remainder, he considers that thirteen are probable and twenty-eight are possible cases of reinfection. Benario says that a diagnosis of reinfection must be based on: (1) a positive clinical diagnosis of the primary lesion, (2) demonstration of Spirochaeta pallida, (3) satellite adenopathy, (4) repeated performance of the Wassermann test, and (5) if possible, recognition of the source of infection. As in all cases in which judgment depends on weighing of evidence rather than on absolute demonstration, another observer might rearrange the cases in his various classes to a certain extent, but on the whole his opinion seems to be just. Since that time the number of reported cases has been considerably increased. The largest series that I have found was reported by White, ${ }^{5}$ who bases his cases on the following proof of reinfection: 1 . In the first attack, spirochetes were found in the chancre or in syphilitic

4. Benario, J.: Samml. swangl. Abhandl. a. d. Geb. d. Dermat. d. Syph., etc. 3:1, 1914.

5. White: Brit. M. J. 2:509 (Oct. 20) 1917. 
lesions, or the blood gave a positive Wassermann reaction (the positive Wasssermann reaction of hereditary syphilis must be excluded). 2. In the second attack, the spirochetes were found in a new chancre, which appeared at a site different. from that of the first chancre, the blood at the same time giving a negative Wassermann reaction. The author presents the histories of ten cases fulfilling these conditions, seen by himself in both attacks in an army hospital, occurring in a series of 10,500 cases of syphilis. He presents eighteen other cases which lack one or more of these requirements-usually either the record of a negative Wassermann reaction at the beginning of the second attack, or the demonstration of the spirochete. The treatment of the first attack usually consisted of eight injections of arsphenamin of $0.3 \mathrm{gm}$. each and from five to seven injections of mercurial cream, each containing 1 grain of mercury, at weekly intervals. The interval between the two attacks was short-seldom over a year. This short interval and scanty treatment throws some doubt on a series otherwise very convincing. It should be remembered, however, that treatment was begun early, and the amount given might well be effective in such cases, while it would be totally inadequate if begun at a later stage of the disease.

A somewhat cursory search of recent literature reveals reports of convincing cases by Jeanselme and Vernes, ${ }^{6}$ Gaston and SanglierLamark, ${ }^{7}$ Milian and Sauphar, ${ }^{8}$ Parounagian, ${ }^{9}$ Sweitzer, ${ }^{10}$ Berent, ${ }^{11}$ Deroide, ${ }^{12}$ Panton and Simpson, ${ }^{13}$ Spangenthal, ${ }^{14}$ Schamberg, ${ }^{15}$ Laurent, ${ }^{16}$ and Saraphi. ${ }^{17}$

A case reported by Lichtenstein ${ }^{18}$ and one reported by Irvine ${ }^{19}$ are probably both second infections, but as neither gives the record of a negative Wassermann reaction at or just before the beginning of the second attack, the evidence of a cure of the first infection is incomplete.

6. Jeanselme and Vernes: Bull. Soc. franç de dermat. et syph. 22:353, 1911.

7. Gaston and Sanglier-Lamark: Bull. Soc. franç. de dermat. et syph. 23: 468, 1912.

8. Milian and Sauphar: Bull. Soc. franç. de dermat. et syph. 24:7, 1913.

9. Parounagian: New York M. J. 103:153 (Jan. 22) 1916.

10. Sweitzer, S. E.: Reinfection in Syphilis, J. A. M. A. 66:1196 (Apri] 15) 1916.

11. Berent: München. med. Wchnschr., Sept. 26, 1916, p. 1408.

12. Deroide: Bull. et mém. Soc. méd. d. hôp. de Par., Ser. 3, 41:603, 1917.

13. Panton and Simpson: Brit. M. J. 1:535 (May 11) 1916.

14. Spangenthal, J.: A Case of Syphilitic Reinfection, J. A. M. A. 71:730

(Aug. 31) 1918.

15. Schamberg: J. A. M. A., Sept. 13, 1919, p. 826.

16. Laurent: Bull. Soc. franç. de dermat. et syph. 27:15, 1920.

17. Saraphi: Ann. d. mal. ven. 15:439, 1920.

18. Lichtenstein: Wien. klin. Wchnschr. 29:1208 (Sept. 21) 1916.

19. Irvine: Brit. M. J. 1:765, 1917. 
In a case reported by Kermorgant, ${ }^{20}$ the diagnosis of the first attacks rests on clinical evidence, and no secondary conditions were observed, except a polymicroadenopathy.

Two cases reported by Goubeau ${ }^{21}$ deserve more than passing notice. The first concerns a soldier who, in August, 1916, presented on the dorsum of the penis a typical chancre in which spirochetes were demonstrated, which healed under treatment with neo-arsphenamin and a silver preparation. On Dec. 13, 1916, the Wassermann reaction was negative, and the patient showed a typical indurated chancre at the extremity of the prepuce, with inguinal adenopathy. He denied exposure since July, and the author therefore believed this to be a pseudo-chancre; but as denial of exposure is not allowed to prevent a diagnosis of chancre in other cases, it should not be allowed to do so in this case. It is to be noted that the two lesions were $2 \mathrm{~cm}$. apart. Goubeau's second case is one of evident reinfection. A man developed a chancre followed by typical secondary conditions in October, 1915. He received intensive mercurial treatment and two courses of arsphenamin of four injections each. There were no further symptoms, and the Wassermann reaction was negative. In November, 1916, after exposure, he developed a new chancre, with satellite adenopathy, followed after six weeks by roseola. It is not stated whether the two chancres were in the same or in different places, but as Fournier ${ }^{3}$ reports cases of pseudo-chancre occurring at sites different from that of the primary lesion, this detail hardly seems to deserve the attention that has been paid to it.

A decision as to the authenticity of any case of reinfection must, in the last analysis, rest on the weighing of probabilities. It must be acknowledged that tertiary lesions which are indistinguishable from chancres were in the same or in different places, but as Fournier by inguinal adenopathy, that it may be followed by an eruption of secondary character, that spirochetes may be demonstrated in the secretion, and that the Wassermann reaction may at the time be negative. Each of these occurrences is rare, and the chance that they should coexist is remote indeed. This coexistence, is however, possible, and as long as only a small number of cases of this nature were known, this explanation might be accepted. When the number of cases multiply, however, some explanation beyond mere chance combination is required. It may be that the occurrence of spirochetes in great numbers in a tertiary lesion would tend to produce satellite adenopathy, and that both would favor the development of relapsing secondary ertption, but we have no evidence of this, and it is hard to reconcile such a view with

20. Kermorgant: Bull. et mém. Soc. méd. d. hôp. de Par., Ser. 3 41:886, 1917.

21. Goubeau: Presse méd. 25:325, 1917. 
the occurrence of a persistent negative Wassermann reaction. When a rapid increase in reported cases of this combination of symptoms is found to coincide with an epoch-making advance in efficacy of treatment, and especially when we note that this treatment has been followed in practically all cases reported, the conclusion is almost inevitable that the occurrence of the syndrome is dependent on the treatment, that is, that these are true cases of reinfection in patients who have been cured. 